Check for updates

Cite this: Phys. Chem. Chem. Phys., 2018, 20, 20334

Received 23rd May 2018,

Accepted 13th July 2018

DOI: $10.1039 / c 8 c p 03274 b$

rsc.li/pccp

\section{Unusual binding-site-specific photophysical properties of a benzothiazole-based optical probe in amyloid beta fibrils $\dagger$}

\author{
N. Arul Murugan, (D) *a Robert Zaleśny ${ }^{(D)}{ }^{b}$ and Hans Ågren (D) ac
}

Optical imaging of amyloid fibrils serves as a cost-effective route for the diagnosis of Alzheimer-like conformational diseases. However, the challenge here is to optimize the binding affinity and photophysical properties of the optical imaging agents in a way specific to certain types of amyloids. In a few occasions it is shown that novel optical imaging agents can be designed to bind to a particular type of amyloid fibril with larger binding affinity and specificity. There is also a recent report on photoluminescent polythiophenes which display photophysical properties that can be used to distinguish the variants or subtypes of amyloids (J. Rasmussen et al., Proc. Natl. Acad. Sci. U. S. A., 2017, 114(49), 13018-13023). Based on a multiscale modeling approach, here, we report on the complementary aspect that the photophysical properties of a benzothiazole based optical probe (referred to as BTA-3) can be specific to the binding sites in the same amyloid fibrils and we attribute this to its varying electronic structure in different sites. As reported experimentally from competitive binding assay studies for many amyloid staining molecules and tracers, we also show multiple binding sites in amyloid fibrils for this probe. In particular, BTA-3 displayed a red-shift in its low-frequency absorption band only in site-4, a surface site of amyloid fibrils when compared to the spectra in water solvent. In the remaining sites, it exhibited a less significant blue shift for the same absorption band.

\footnotetext{
${ }^{a}$ Department of Theoretical Chemistry and Biology, School of Engineering Sciences in Chemistry, Biotechnology and Health, KTH Royal Institute of Technology, S-106 91, Stockholm, Sweden. E-mail: murugan@kth.se

${ }^{b}$ Department of Physical and Quantum Chemistry, Faculty of Chemistry, Wrockaw University of Science and Technology, Wyb. Wyspianskiego 27, PL-50370 Wrockaw, Poland

${ }^{c}$ Department of Physics and Astronomy, Uppsala University, Box 516, SE-751 20 Uppsala, Sweden

$\dagger$ Electronic supplementary information (ESI) available: Description of computational methods: molecular docking, molecular dynamics, Car-Parrinello hybrid QM/MM molecular dynamics, absorption spectra calculations using TD-DFT/MM and CC/MM approaches, free energy calculations using the molecular mechanics/ generalized Born surface area approach. Fig. S1 showing the frontier molecular orbitals involved in the first two low energy excitations. Table 1 giving the spatial overlap parameter computed to justify the use of the B3LYP level of theory to analyze the absorption spectra of BTA-3. Fig. S2 showing the molecular electrostatic potential computed for the whole fibril. See DOI: $10.1039 / \mathrm{c} 8 \mathrm{cp} 03274 \mathrm{~b}$
}

\section{Introduction}

A promising future for clinical diagnostics refers to the use of blood, sweat, cerebrospinal and other body fluids or even breath to detect diseases at their early stages. ${ }^{1,2}$ Significant advances in the diagnosis of many diseases have been made, thanks to optical imaging agents that display characteristic photophysical properties specific to a disease biomarker. Moreover, the development and sophistication in instrumentation and the ease with which we can record fluorescence and two-photon spectra are factors contributing to the observed progress. The clinical diagnostics of a number of diseases, using body fluids from human subjects, is possible because they carry disease-specific biomarkers and these can be characterised quantitatively using certain optical staining agents. Congo red and thioflavin-T staining remains to be the most popular example for detecting amyloids in a given sample. ${ }^{3,4}$ The red shift of the absorption wavelength observed for these staining agents is a diagnostic parameter for the presence of amyloids. The enhancement in their fluorescence intensity directly correlates with the amount of the amyloid. Furthermore, time-dependent fluorescence measurements can be used to estimate the rate of fibrillization.

The success of diagnosis heavily relies on the protocols for the efficient estimation of biomarkers that are expressed in the early stage of diseases. Micro RNAs, exosomes, different proteins, amyloid oligomers, and fibrils are the potential biomarkers for cancer, diabetes, Alzheimer's and Parkinson's diseases, to name a few. ${ }^{5}$ The main problem is that amyloid fibrils, a class of biomacromolecular aggregates with richer beta sheet contents, appear to be connected to many diseases such as Alzheimer's, Parkinson's and Huntington's diseases, diabetes mellitus type 2 and amyotrophic lateral sclerosis. The fibrils of amyloid beta, tau, $\alpha$-synuclein, amylin, prions and Huntington proteins are all examples of macromolecular aggregates that are associated with these diseases generally referred to as "conformational diseases". Due to the similarity in their secondary structure, by and large, many of the amyloid staining 
compounds bind to these fibrils non-specifically thus making it difficult to distinguish between different types of amyloidrelated diseases. Hence, a major challenge in diagnostics is to design amyloid-specific optical probes.

Recently, a few promising optical probes have been experimentally synthesised that can bind specifically to one type of amyloid fibrils and can even distinguish between the mutant variants or subtypes of the same amyloid. ${ }^{6}$ For example, the coumarin-quinoline (CQ) conjugate-based turn-on near-infrared (NIR) fluorescence probe has been reported to bind to amyloid beta plaques specifically but not to other fibrils like tau, alphasynuclein and islet amyloid polypeptide. ${ }^{7}$ Similarly, a BODIPYbased BD-tau probe has been reported to display tau-specific fluorescence properties and has shown comparably lower binding to other fibrils of amylin and amyloid beta proteins. ${ }^{8}$ Aminonaphthalene 2-cyanoacrylate probes, which are specific to amyloid beta fibrils over prion fibrils, have also been reported. ${ }^{9}$ Recently, certain luminescent conjugated polythiophenes have been demonstrated to show variation in optical properties depending on the subtypes of the amyloid beta fibrils. ${ }^{6}$ Even though the experimental studies demonstrate that amyloid-specific staining agents can be developed, the computer simulations are the only way to systematically and rationally design such compounds.

Currently we have access to a huge chemical space ${ }^{10,11}$ and recently new structures for many amyloid fibrils have become available, thanks to the cryogenic electron microscopy technique. ${ }^{12,13}$ By using these structures in computer modeling, one can design optical probes that can specifically bind to various types of amyloid fibrils. However, this requires the knowledge about the local microstructure in different binding sites and the strategy will be to optimize the binding to core sites as this will have a fibril-specific microenvironment rather than to surface sites. In addition, there is also a need to design optical probes that can display photophysical properties specific to a given binding site. To contribute to this subject, we investigate here various binding sites of the benzothiazole-based optical probe (hereafter referred to as BTA-3; for the molecular structure see Fig. 1) in amyloid fibrils and its site-specific changes in the molecular structure and optical properties. Recent experimental reports indicate that benzothiazole derivatives can be used as optical probes for the detection of amyloid beta and $\alpha$-synuclein aggregates. ${ }^{14}$ These molecules could thus serve as an excellent testbed for the ability of current in silico methods for modeling both the binding profile and amyloid-specific photophysical properties. Here we compare the very viable TD-DFT (timedependent density functional theory) approach with a high level $a b$ initio RI-CC2 (resolution-of-identity coupled-cluster CC2) method, as the validation of the former. In both cases, we apply multiscale quantum mechanics molecular mechanics embedding, in particular an electrostatic embedding, ${ }^{15,16}$ referred to as

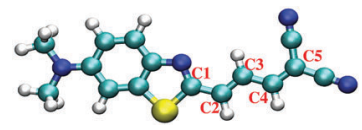

Fig. 1 Molecular structure of BTA-3.
TD-DFT/MM and RI-CC2/MM, respectively, to account for the effect of a large inhomogeneous microenvironment on the optical properties of the BTA-3 probe. The present study thus aims to contribute to the subject by pinpointing how the binding of benzothiazole probes to fibrils can transform their photophysical properties in terms of a shift in absorption wavelength and in their oscillator strengths and then can be used to single out the preferred binding sites. This, in turn, can be vital information for designing new ligands with optimal properties for specific amyloid aggregates.

We have employed various computational approaches in a sequential way to address the binding profile of BTA-3 in an amyloid beta fibril, its relative free energy of binding in different sites, the stability of the complex structure, the binding-site-specific electronic and molecular structure and finally the binding-site-specific optical properties. The amyloid beta fibril itself is known to exist in different polymorphic forms and here we have used the recently reported amyloid beta fibril (PDB id is 5OQV) for a full length (having 42 residues) peptide fragment. ${ }^{13}$

\section{Computational details}

For the calculation of the one-photon absorption properties of BTA-3 in different binding sites of amyloid fibrils we have employed an integrated approach including molecular docking, molecular dynamics, Car-Parrinello hybrid quantum mechanics/ molecular mechanics (QM/MM) molecular dynamics and hybrid $\mathrm{QM} / \mathrm{MM}$ response calculations. Molecular docking has been used to identify various binding sites of BTA-3 in amyloid beta fibrils. The stability of the fibril:BTA-3 complex has been studied using molecular dynamics simulations. The fibril-induced changes in the molecular structure and electronic structure of BTA-3 have been studied using hybrid QM/MM MD. The optical properties of BTA-3 in different binding sites of amyloid fibrils have been studied using TD-DFT/MM and RI-CC2/MM which describe the interaction between the fibril and BTA-3 using an electrostatic embedding scheme. A similar combination of approaches has been used in our previous studies to model the optical properties of molecules bound to biological targets..$^{7,17,18}$ We have also computed the optical spectra of a BTA-3 molecule in water for comparing its spectra in a fibril environment. So, separate molecular dynamics, hybrid QM/MM MD followed by TD-DFT/MM and RI-CC2/MM calculations were performed for a single BTA-3 molecule in water. In order to estimate the binding free energies of BTA-3 in different binding sites of amyloid fibrils molecular mechanicsgeneralized Born surface area based calculations were performed for the configurations obtained from molecular dynamics simulations. In the ESI, $\dagger$ we describe in detail various methods employed in this study.

\section{Results and discussion}

Based on the molecular docking studies, we find that BTA-3 binds to at least four different binding sites as shown in Fig. 2. 


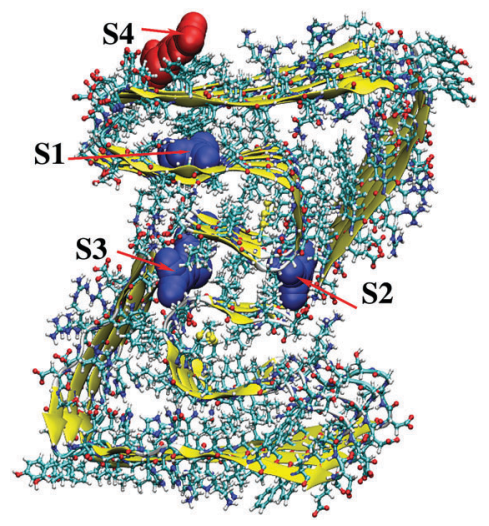

Fig. 2 Various binding sites of a BTA-3 probe in amyloid beta proto fibrils.

It follows from this figure that three of them are core sites and among these, two of them are in the interfacial region of pentamer and tetramer units while the first one is completely localised on the pentamer unit. Fourth one appears on the surface. The binding energies computed from molecular docking for BTA-3 in site-1, site-2, site-3 and site-4 are $-11.0,-8.2,-8.1$ and $-7.6 \mathrm{kcal} \mathrm{mol}^{-1}$, respectively. The inhibition constants are $8.7 \mathrm{nM}$ for site- 1 while $2.5 \mu \mathrm{M}$ for site- 4 . The experimental binding affinity has been reported to be $140 \mathrm{nM}$ suggesting that it may be an average binding affinity over various binding sites. ${ }^{14}$ The experimental study by Watanabe et al. has not attempted to address the multiple binding sites available for BTA-3 in the amyloid fibril even though this has been demonstrated for amyloid specific tracers using competition binding assay experiments. ${ }^{19-22}$ As it has been reported for other staining agents and tracers, there are at least four high affinity binding sites available for BTA-3 in the amyloid fibril. The molecular docking studies suggest that the core sites are slightly preferable than the surface site. However, the molecular docking involves a single fibril structure and does not account for sampling over various configurations. In order to further confirm the relative binding affinities of BTA-3 in different sites, we present the binding free energies based on an MM-GBSA approach in Table 1. This also confirms that site- 1 is the one associated with larger binding affinity while the binding affinity for different sites follows the sequence: site- $1>$ site- $2>$ site- $3>$ site-4. Even though the core sites are energetically preferable, the molecules need to cross the water:fibril interfacial region which may contribute to kinetic barriers. But assessing the surface sites may be easier for a probe molecule. Moreover, in a matured fibril, there are numerous surface sites while the core sites are limited. So, the photophysical properties may have major contributions from surface sites even though they are not the energetically most favorable binding sites.

We have computed the absorption spectra of BTA-3 in different binding sites. Since site- 2 and site- 3 are essentially the same (interfacial binding sites), the calculations were performed only for site- 2 . As a reference the absorption spectra of BTA-3 have also been computed in water solvent. We employed time-dependent density functional theory/molecular mechanics
Table 1 Binding free energies of BTA-3 in different binding sites of amyloid beta fibrils using an MM-GBSA approach. The unit for binding energy is $\mathrm{kcal} \mathrm{mol}^{-1}$

\begin{tabular}{llrlll}
\hline Site & $\Delta E_{\mathrm{vdW}}$ & $\Delta E_{\text {elec }}$ & $\Delta G_{\mathrm{GB}}$ & $\Delta G_{\mathrm{SA}}$ & $\Delta G_{\text {binding }}$ \\
\hline Site-1 & -60.8 & -11.7 & 32.0 & -5.9 & -46.5 \\
Site-2 & -46.2 & -2.7 & 29.7 & -5.2 & -24.4 \\
Site-3 & -37.9 & -13.2 & 31.5 & -5.2 & -24.8 \\
Site-4 & -28.9 & -47.5 & 58.6 & -3.5 & -21.3
\end{tabular}

theory with two different functionals namely B3LYP and CAMB3LYP. The absorption band in the spectra obtained from the latter was blue-shifted, and, because of the better agreement with experiment, the B3LYP functional (with the TZVP basis set) was used for further analysis of the optical properties. The use of the B3LYP level of theory for the discussion on the absorption spectra of BTA-3 in different microenvironments is justified based on the following arguments. As we will see in the discussion below, the B3LYP/MM based absorption spectra were quantitatively in closer agreement with $\mathrm{CC} 2 / \mathrm{MM}$ based results when compared to CAM-B3LYP/MM based results. Furthermore, as shown in the $\mathrm{ESI}, \dagger$ the spatial overlap calculated for the low energy excitation is 0.65 which suggests that the B3LYP level of theory is capable of describing this type of excitation. ${ }^{23}$ It follows from the results shown in Fig. 3a that indeed the absorption spectra of a BTA-3 probe is rather site-specific. The absorption maximum for BTA-3 in water solvent is $587 \mathrm{~nm}$ ( $c f$. Table 2). In binding sites 1 and 2, the absorption band maximum in the spectra is blue-shifted by a few $\mathrm{nm}(7 \mathrm{~nm})$ when compared to the spectra in water solvent ( $c f$. Table 2). However, in site-4 the band in spectra was red-shifted by $68 \mathrm{~nm}$ when compared to the spectra in water. In addition to the shift in the absorption maximum, there is also a noticeable increase in the oscillator strength (absorption intensity) for BTA-3 when it is bound to the amyloid fibril. In particular, the increase in intensity is much larger for site- 1 and site-2. It is also notable that there is another band appearing around $411 \mathrm{~nm}$ in water solvent which exhibits a red shift at all sites and the amount of

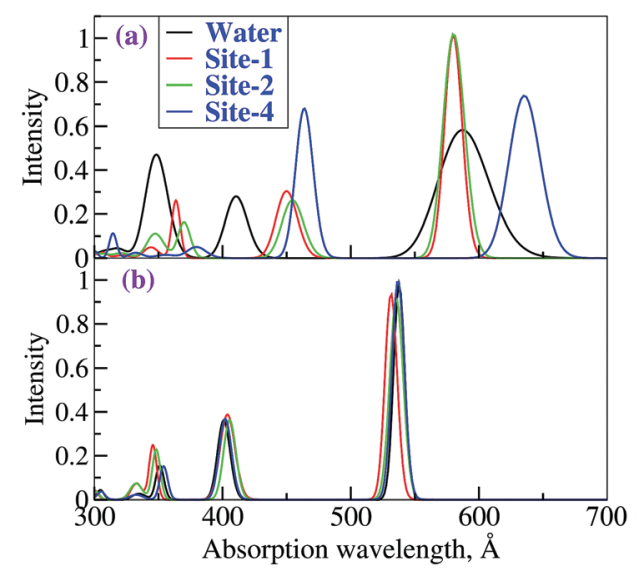

Fig. 3 (a) Absorption spectra of BTA-3 in three different sites using sampling with hybrid QM/MM molecular dynamics. The one-photon absorption spectra were calculated using a TD-DFT/MM approach at the B3LYP/TZVP level of theory. (b) The results for one-photon absorption spectra without including solvent or a fibril environment. 
Table 2 Position of absorption band maxima (in [nm]) and oscillator strengths computed using a TD-DFT/MM and RI-CC2/MM level of theory

\begin{tabular}{llccc}
\hline System & Water & Site-1 & Site-2 & Site-4 \\
\hline TD-DFT/MM & & & & \\
Excitation-1 & $587(0.6)$ & $580(1.0)$ & $580(1.0)$ & $635(0.7)$ \\
Excitation-2 & $410.6(0.3)$ & $450.0(0.3)$ & $454.6(0.3)$ & $463.6(0.7)$ \\
& & & & \\
RI-CC2/MM & & & & \\
Excitation-1 & $641(1.3)$ & $639(1.3)$ & $642(1.3)$ & $718(1.2)$ \\
Excitation-2 & $386(0.3)$ & $393(0.2)$ & $396(0.2)$ & $425(0.5)$
\end{tabular}

shift is found to be specific to the binding site. The red shift in the second band is 40,45 and $55 \mathrm{~nm}$ for site-1, site-2 and site- 4 , respectively. We have also analyzed the nature of excitations corresponding to the first two bands in the absorption spectra. The first one is dominated by single-electron HOMO $\rightarrow$ LUMO transition and it is associated with a significant electronic density shift from the phenyl ring towards cyano groups. The second one is dominated by HOMO-1 $\rightarrow$ LUMO type excitation (refer to Fig. S1 of the ESI $\dagger$ to see MOs involved in these excitations).

The site-specific absorption spectra of BTA-3 in amyloid beta fibrils were also computed using the sophisticated RI-CC2/MM level of theory and the results confirm the B3LYP-based results. The red shift at site- 4 is found to be $78 \mathrm{~nm}$ at the coupledcluster level of theory. However, the shifts of the absorption maximum in site- 1 and site- 2 are now less pronounced, as predicted at the RI-CC2/MM level of theory. The amyloidspecific red shift in the second band is also less pronounced for site- 1 and site- 2 but the calculated red shift is significant for site-4 (approximately $39 \mathrm{~nm}$ ).

The aforementioned red shift in the first intense band in the absorption spectra has been reported for many amyloid staining compounds including Congo red and thioflavin-T (THT), to name a few, and it has been used as a diagnostic parameter for amyloids. $^{24,25}$ The red shift in the absorption spectra of these compounds has been attributed to the planarization of the molecules in the fibril-like environment. ${ }^{24,25}$ In the case of BTA-3 it is not clear whether such amyloid-induced structural changes contribute to the spectral shift. It appears that the benzothiazole, spacer group and the diacyano groups in BTA-3 should maintain a planar conformation independent of the nature of the microenvironment due to energetics reasons as the hyperconjugation stabilizes such geometries. However, there are possible changes in the bond-length alternation values which are reported to be sensitive to the dielectric nature of the environment. ${ }^{26,27}$

For example, we have reported previously that the bond length alternation values for polymethines and merocyanines can vary significantly, i.e. from positive to negative values, depending on the environment. ${ }^{26,27}$ In other words, these families of molecules can adopt neutral-like (charge separation is minimum), cyanine-like or zwitterionic (charge separation is maximum) structures depending on the solvent environment which is then reflected in their dielectric-specific optical properties. ${ }^{26,27}$ To assess whether the spectral shifts are due

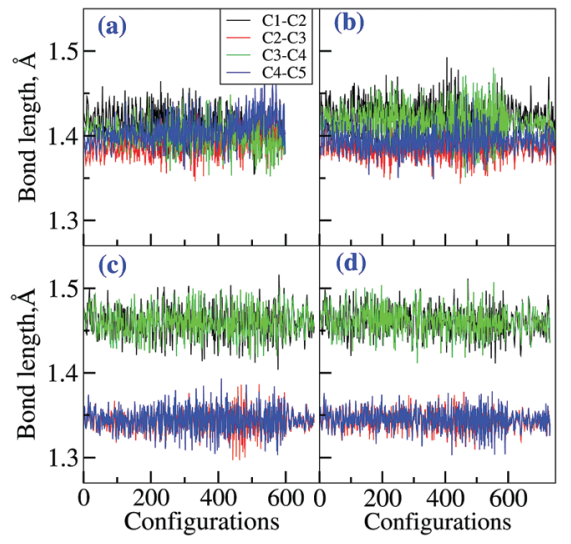

Fig. 4 Time evolution of instantaneous geometrical parameters of BTA-3 (a) in water, (b) in site-1, (c) in site-2 and (d) in site-4 of amyloid beta fibrils.

to changes in the ground state molecular structure or electronic structure, we have also computed the absorption spectra of BTA-3 molecules (in different environments like water, and in different binding sites of amyloid fibrils) using the TD-DFT and RI-CC2 levels of theory. Note that the interaction with the environment, which is accounted for by the electrostatic embedding in the aforementioned results has been discarded in these calculations. So, any significant changes in the photophysical properties and spectral shifts observed based on the results of these calculations should be accounted for by the environment-induced structural changes in BTA-3. The results are shown in Fig. 3b. As can be seen, the absorption maximum for the first band appears at $530 \mathrm{~nm}$ in all the cases suggesting that the spectral shifts in BTA-3 in different sites cannot be attributed to any fibril- or solvent-induced structural changes.

In order to rationalize the spectral shift of BTA-3 in different sites, we have analysed the molecular structure of BTA-3 in water and chloroform solvents and in different binding sites of the amyloid fibrils. The time evolution of bond length values for the four bonds along the conjugation pathway namely $\mathrm{C} 1-\mathrm{C} 2, \mathrm{C} 2-\mathrm{C} 3, \mathrm{C} 3-\mathrm{C} 4$ and $\mathrm{C} 4-\mathrm{C} 5$ are shown in Fig. 4 and their time-averaged values are presented in Table 3. As can be seen, the bond length values and the molecular geometry are comparable for the cases of BTA-3 in water and in site- 1 . Note that the molecular geometries in site-2, site-4 and in chloroform are comparable. Even though the molecular geometries of the spacer group are quite different in these cases, i.e. in water and in different binding sites of the amyloid beta fibrils, the spectra appeared the same, thus suggesting that the shifts in spectra cannot be attributed to this effect. The results are slightly unexpected that even though the molecular structure

Table 3 Average bond lengths along the conjugation pathway for BTA-3 (calculated using trajectories from hybrid QM/MM molecular dynamics)

\begin{tabular}{llllll}
\hline Bonds & Water & Site-1 & Site-2 & Site-4 & $\mathrm{CHCl}_{3}$ \\
\hline C1-C2 & 1.414 & 1.424 & 1.460 & 1.461 & 1.427 \\
C2-C3 & 1.391 & 1.386 & 1.342 & 1.343 & 1.379 \\
C3-C4 & 1.401 & 1.417 & 1.460 & 1.461 & 1.417 \\
C4-C5 & 1.407 & 1.394 & 1.344 & 1.345 & 1.386
\end{tabular}


Table 4 Average group charges and molecular dipole moments for BTA-3 in solvents as well as in different binding sites of amyloid fibrils as calculated from hybrid QM/MM molecular dynamics. The charges are in electronic unit and dipole moments are in debye unit. The charges are averaged over the ESP charges computed for each of the configurations of the trajectory and so are averaged approximately over 60000 configurations

\begin{tabular}{|c|c|c|c|c|c|}
\hline$q, e$ & Water & Site-1 & Site-2 & Site-4 & $\mathrm{CHCl}_{3}$ \\
\hline \multicolumn{6}{|c|}{ Group charges } \\
\hline $\mathrm{ESP}_{\mathrm{Grp} 1}$ & 0.388 & 0.320 & 0.310 & 0.371 & 0.262 \\
\hline $\mathrm{ESP}_{\mathrm{Grp} 2}$ & -0.049 & -0.098 & -0.041 & -0.018 & -0.063 \\
\hline $\mathrm{ESP}_{\mathrm{Grp} 3}$ & 0.282 & 0.277 & 0.199 & 0.262 & 0.178 \\
\hline $\mathrm{ESP}_{\mathrm{Grp} 4}$ & -0.618 & -0.495 & -0.467 & -0.611 & -0.372 \\
\hline \multicolumn{6}{|c|}{ Dipole moment } \\
\hline$\mu$, Debye & 12.29 & 9.63 & 9.32 & 12.00 & 7.10 \\
\hline
\end{tabular}

of BTA-3 is different in binding sites, this does not contribute to the observed spectral shifts. Furthermore, we have analysed the group charges and molecular dipole moments of BTA-3 in different solvent environments and in different binding sites of amyloid fibrils. The results are presented in Table 4. Grp1 refers to the dimethyl amino group and Grp2, Grp3 and Grp4, respectively, refer to the benzothiazole group, $-\mathrm{CH}=\mathrm{CH}-\mathrm{CH}=$ and dicyanomethyl groups. The group charges and molecular dipole moments were computed using the electrostatic potential fitted charges available for each atom in BTA-3 in the hybrid $\mathrm{QM} / \mathrm{MM} \mathrm{MD}^{28}{ }^{28}$ As can be seen, the molecular dipole moment and group charges for BTA-3 in site- 1 and site- 2 are comparable and explain why the spectra in these sites for BTA-3 were similar. In addition, the charge separation and dipole moment for BTA-3 in site 1 , site 2 and in chloroform are the least when compared to those for BTA-3 in site-4 and in water solvent. In other words BTA-3 in site 4 and in water solvent adopts a charge separated form when compared to BTA-3 in site 1, site 2 and in chloroform solvent. The red shift of absorption band for BTA-3 in site 4 when compared to in water can be easily explained. As we learn from the literature and from our own experience, ${ }^{26,27}$ the ground state of the charge separated form is stabilized largely by more polar solvents compared to a less polar microenvironment leading to a red shift in the spectra in the latter case. Overall, the difference in the electronic structure of BTA-3 in different binding sites is responsible for the variation in spectral shifts. Even though, the solvent influence in the electronic and molecular structure of a molecular probe can be easily appreciated, it is generally not known that different binding sites in amyloid beta fibrils can alter these properties substantially. Here we make a report of this observation that the molecular and electronic structure of BTA-3 is specific to binding sites in amyloid fibrils and in addition the latter quantity dictates on the nature of the spectral shift in different binding sites. It has been reported in the case of human aldose reductase that the electric field generated at the catalytic site can be even influenced by a single point mutation and can lead to a shift in the infrared absorption spectra of a nitrile group based IR probe. ${ }^{29}$ So it is not surprising that the local microenvironment in different binding sites can be different and this is dictated by the specific sequence of amino acids constituting the binding site. The results of recent measurements of the optical properties of certain luminescent polythiophenes have shown that their photophysical properties are dependent on the specific mutations which again suggest that single mutation can influence the photophysical properties of the polythiophene optical probe ${ }^{6}$ Hence, it comes as no surprise that if there are multiple binding sites, then the dielectric nature of the local microenvironment can be different depending on the constituent amino acids. So, the sitespecific photophysical properties could be attributed to such differences in the microenvironment. In order to support this explanation we have plotted the electrostatic potential for the whole amyloid fibril and the result is given in the ESI. $\dagger$ The red color shows the distribution of negative charges and the blue color shows the domains with the depleted charges. As can be seen, the electrostatic potential appears to be site-specific and this leads to differential stabilization of the ground and excited states of BTA-3 in different sites. Overall, the absorption spectra of the BTA-3 probe are specific to the binding-site microenvironment.

\section{Conclusions}

To sum up, we report several possible binding sites for the BTA-3 optical probe in amyloids beta fibrils using molecular docking studies. Similar to earlier reports on amyloid staining molecules and tracers based on competition binding assay experiments, we find that there are multiple binding sites available for BTA-3. The relative binding affinities in the different sites have been assessed using molecular dynamics and the MM-GBSA approach and based on these results we suggest that the core sites are slightly preferred over the surface sites. Furthermore, the site specific changes in the molecular structure and electronic structure of BTA-3 when compared to aqueous conditions have been demonstrated using hybrid QM/MM molecular dynamics simulations. The molecular and electronic structures of BTA-3 are found to be sensitive to the local microenvironment which we have also observed in the case of other optical probes, like merocyanine and polymethine. ${ }^{26,27,30}$ By using TD-DFT/MM and RI-CC2/MM approaches we calculated the absorption spectra of the BTA-3 molecule in different sites of the amyloid fibrils. The generally reported red shift of an absorption band is seen only when BTA-3 is bound to site- 4 which is a surface site. The changes in the spectral properties are attributed to the variation in the dielectric nature of the microenvironment in different binding sites. Even though the molecular geometries display significant changes in the different sites, these do not noticeably contribute to the spectral shift. Rather the changes in the electronic structure i.e. the charge distribution dictated by the external solvent- and bio-environment controls the photophysical properties of BTA-3 in different sites. The study shows that the photophysical properties of a certain optical probe can be specific to binding sites in biomacromolecular targets. Although the solvent polarity-specific optical properties of such molecules have been successfully addressed using integrated approaches, this is the first report showing that probes can also be used to sense the variation in the dielectric nature of the binding sites. This result does, however, not come as a surprise given that certain polythiophenebased luminescent probes have been shown to display optical 
properties specific to subtypes or variants of amyloids. A possible explanation could be derived from our study that the local binding site microenvironment is altered by the mutations which, in turn, alters the optical properties of these polythiophene-based probes. ${ }^{6}$

\section{Conflicts of interest}

There are no conflicts to declare.

\section{Acknowledgements}

The authors acknowledge support from the Swedish Foundation for Strategic Research (SSF) through the project "New imaging biomarkers in early diagnosis and treatment of Alzheimer's disease" and the support from SLL through the project "Biomolecular profiling for early diagnosis of Alzheimer's disease”. This work was supported by the grants from the Swedish Infrastructure Committee (SNIC) for the projects "Multiphysics Modeling of Molecular Materials" (SNIC2015-16-10) and "In silico Diagnostic Probes Design” (SNIC2015-1-486). R. Z. acknowledges financial support from the Polish National Science Centre (Grant No. 2015/ 19/B/ST4/01881). The calculations were performed in part at the Wroclaw Center for Networking and Supercomputing.

\section{References}

1 M. Pitschke, R. Prior, M. Haupt and D. Riesner, Nat. Med., 1998, 4, 832-834.

2 A. Foutz, B. S. Appleby, C. Hamlin, X. Liu, S. Yang, Y. Cohen, W. Chen, J. Blevins, C. Fausett and H. Wang, et al., Ann. Neurol., 2017, 81, 79-92.

3 E. Benditt, N. Eriksen and C. Berglund, Proc. Natl. Acad. Sci. U. S. A., 1970, 66, 1044-1051.

4 T. Ban, D. Hamada, K. Hasegawa, H. Naiki and Y. Goto, J. Biol. Chem., 2003, 278, 16462-16465.

5 T. M. Marques, H. B. Kuiperij, I. B. Bruinsma, A. van Rumund, M. B. Aerts, R. A. Esselink, B. R. Bloem and M. M. Verbeek, Mol. Neurobiol., 2017, 54, 7736-7745.

6 J. Rasmussen, J. Mahler, N. Beschorner, S. A. Kaeser, L. M. Häsler, F. Baumann, S. Nyström, E. Portelius, K. Blennow and T. Lashley, et al., Proc. Natl. Acad. Sci. U. S. A., 2017, 114, 13018-13023.

7 K. Rajasekhar, N. Narayanaswamy, N. A. Murugan, K. Viccaro, H.-G. Lee, K. Shah and T. Govindaraju, Biosens. Bioelectron., 2017, 98, 54-61.

8 S. Lim, M. M. Haque, D. Su, D. Kim, J.-S. Lee, Y.-T. Chang and Y. K. Kim, Chem. Commun., 2017, 53, 1607-1610.
9 K. Cao, M. Farahi, M. Dakanali, W. M. Chang, C. J. Sigurdson, E. A. Theodorakis and J. Yang, J. Am. Chem. Soc., 2012, 134, 17338-17341.

10 C. Lipinski and A. Hopkins, Nature, 2004, 432, 855.

11 C. M. Dobson, Nature, 2004, 432, 824.

12 A. W. Fitzpatrick, B. Falcon, S. He, A. G. Murzin, G. Murshudov, H. J. Garringer, R. A. Crowther, B. Ghetti, M. Goedert and S. H. Scheres, Nature, 2017, 547, 185-190.

13 L. Gremer, D. Schölzel, C. Schenk, E. Reinartz, J. Labahn, R. B. Ravelli, M. Tusche, C. Lopez-Iglesias, W. Hoyer, H. Heise, D. Willbold and G. F. Schröder, Science, 2017, 358, 116-119.

14 H. Watanabe, M. Ono, T. Ariyoshi, R. Katayanagi and H. Saji, ACS Chem. Neurosci., 2017, 8, 1656-1662.

15 J. M. Olsen and J. Kongsted, Adv. Quantum Chem., 2011, 61, 107-143.

16 J. M. Olsen, K. Aidas and J. Kongsted, J. Chem. Theory Comput., 2010, 6, 3721-3734.

17 N. A. Murugan, J. Kongsted, Z. Rinkevicius and H. Ågren, Phys. Chem. Chem. Phys., 2012, 14, 1107-1112.

18 N. A. Murugan, J. M. H. Olsen, J. Kongsted, Z. Rinkevicius, K. Aidas and H. Ågren, J. Phys. Chem. Lett., 2013, 4, 70-77. 19 H. Levine, III, Amyloid, 2005, 12, 5-14.

20 A. Lockhart, L. Ye, D. B. Judd, A. T. Merritt, P. N. Lowe, J. L. Morgenstern, G. Hong, A. D. Gee and J. Brown, J. Biol. Chem., 2005, 280, 7677-7684.

21 R. Ni, P.-G. Gillberg, A. Bergfors, A. Marutle and A. Nordberg, Brain, 2013, 136, 2217-2227.

22 R. Kawai, M. Araki, M. Yoshimura, N. Kamiya, M. Ono, H. Saji and Y. Okuno, ACS Chem. Neurosci., 2018, 957-966.

23 M. J. G. Peach, P. Benfield, T. Helgaker and D. J. Tozer, J. Chem. Phys., 2008, 128, 044118.

24 T. Miura, C. Yamamiya, M. Sasaki, K. Suzuki and H. Takeuchi, J. Raman Spectrosc., 2002, 33, 530-535.

25 L. S. Wolfe, M. F. Calabrese, A. Nath, D. V. Blaho, A. D. Miranker and Y. Xiong, Proc. Natl. Acad. Sci. U. S. A., 2010, 107, 16863-16868.

26 N. A. Murugan, K. Aidas, J. Kongsted, Z. Rinkevicius and H. Ågren, Chem. - Eur. J., 2012, 18, 11677-11684.

27 N. A. Murugan, J. Kongsted, Z. Rinkevicius, K. Aidas and H. Ågren, J. Phys. Chem. B, 2010, 114, 13349-13357.

28 A. Laio, J. VandeVondele and U. Rothlisberger, J. Chem. Phys., 2006, 116, 6941.

29 I. T. Suydam, C. D. Snow, V. S. Pande and S. G. Boxer, Science, 2006, 313, 200-204.

30 N. A. Murugan, J. Kongsted, Z. Rinkevicius and H. Ågren, Proc. Natl. Acad. Sci. U. S. A., 2010, 107, 16453-16458. 